Ophthalmologe 2022 $\cdot 119: 721-729$

https://doi.org/10.1007/s00347-022-01574-x

Eingegangen: 8. Juli 2021

Überarbeitet: 1. Januar 2022

Angenommen: 3. Januar 2022

Online publiziert: 2. Februar 2022

(c) Der/die Autor(en) 2022

\section{Binokulares Sehtraining bei Hochleistungssportlern}

\title{
Zusammenfassung
}

Hintergrund und Fragestellung: Gutes Stereosehen scheint bei Hochleistungssportlern und im Alltag eine wichtige Rolle zu spielen. Wir untersuchten die Fragestellung, ob sich das Stereosehen bei Sportlern mit bereits guter Stereosehschärfe durch ein digitales Sehtraining noch verbessern kann.

Studiendesign und Untersuchungsmethoden: $N=31$ männliche und weibliche Tennisspieler/innen absolvierten innerhalb 6 Wochen mindestens 6 Trainingseinheiten mit je 192 stereoskopischen Einzeltests mit variablem Schwierigkeitsgrad von 15 bis 300 Bogensekunden. Die Trainingsstimuli waren dynamischer, die Teststimuli statischer Natur. Zur Darstellung der Reaktionszeitverbesserung in Abhängigkeit des Schwierigkeitsgrades wurde der Parameter "ReSt" (Reaktionszeitzuwachs pro Stereodisparitätsabnahme) bestimmt.

Ergebnisse: Die Reaktionszeit bei Stimuli von 15 Bogensekunden verkürzte sich durch das Sehtraining im Mittel signifikant von 3,9s auf 1,6s (59\%). Die Korrektheit der Versuche bei 30 Bogensekunden steigerte sich im Mittel signifikant um $23 \%$.

Diskussion: Die beobachtete Verbesserung der Reaktionszeit im Sehtraining führte nicht zur Abnahme der Korrektheit bei der Beantwortung der visuellen Fragen und stellt damit insgesamt eine Verbesserung der Stereosehfähigkeit dar.

Schlussfolgerung: Durch das digitale Sehtraining verbesserte sich das stereoskopische Sehen signifikant hinsichtlich Stereogrenzwinkel, Reaktionszeit und Korrektheit.

\section{Schlüsselwörter}

Stereosehen · Sehtraining · Tiefenwahrnehmung $\cdot$ Sport · Kognitionszeit

\section{Hintergrund und Fragestellung}

Eine Verschlechterung des visuellen Systems führt ursachenunabhängig zu einer Verminderung der funktionellen Aktivitäten und Lebensqualität [1]. Voraussetzung für ausgezeichnetes Sehen ist gutes 3-dimensionales Sehen. Es setzt eine gute Sehschärfe und Fusion der Bildeindrücke beider Augen voraus. So haben ältere Autofahrer mit verminderter Stereosehschärfe und Sehschärfe ein erhöhtes Unfallrisiko [2]. Bei alleiniger Verminderung der Sehschärfe bleibt hingegen das Unfallrisiko konstant. Auch das allgemeine Sturzrisiko steigt bei Personen mit verminderter Stereosehfähigkeit an [3]. Gute Stereosehschärfe scheint nicht nur im Alltag, son- dern auch im Hochleistungssport wichtig zu sein [4].

Spieler aus der "National Football League" in sog. "skilled positions" (Positionen, die am unmittelbarsten dafür verantwortlich sind, dass Punkte erzielt oder verhindert werden) weisen eine schnellere visuelle Reaktionszeit auf [5]. Bei Basketballspielern zeigte sich eine signifikante Korrelation zwischen dynamischer Sehschärfe und Treffsicherheit der Körbe [6]. Ohne binokulare Sehfähigkeit kommt es zu einer starken Abnahme z.B. der Treffsicherheit bei Freiwürfen [7].

Stereosehschärfe wird definiert als der kleinste noch erkannte Distanzunterschied zweier Objekte, der zu einer räumlichen Tiefenwahrnehmung führt, gemessen in Bogensekunden. Je kleiner der noch er- 


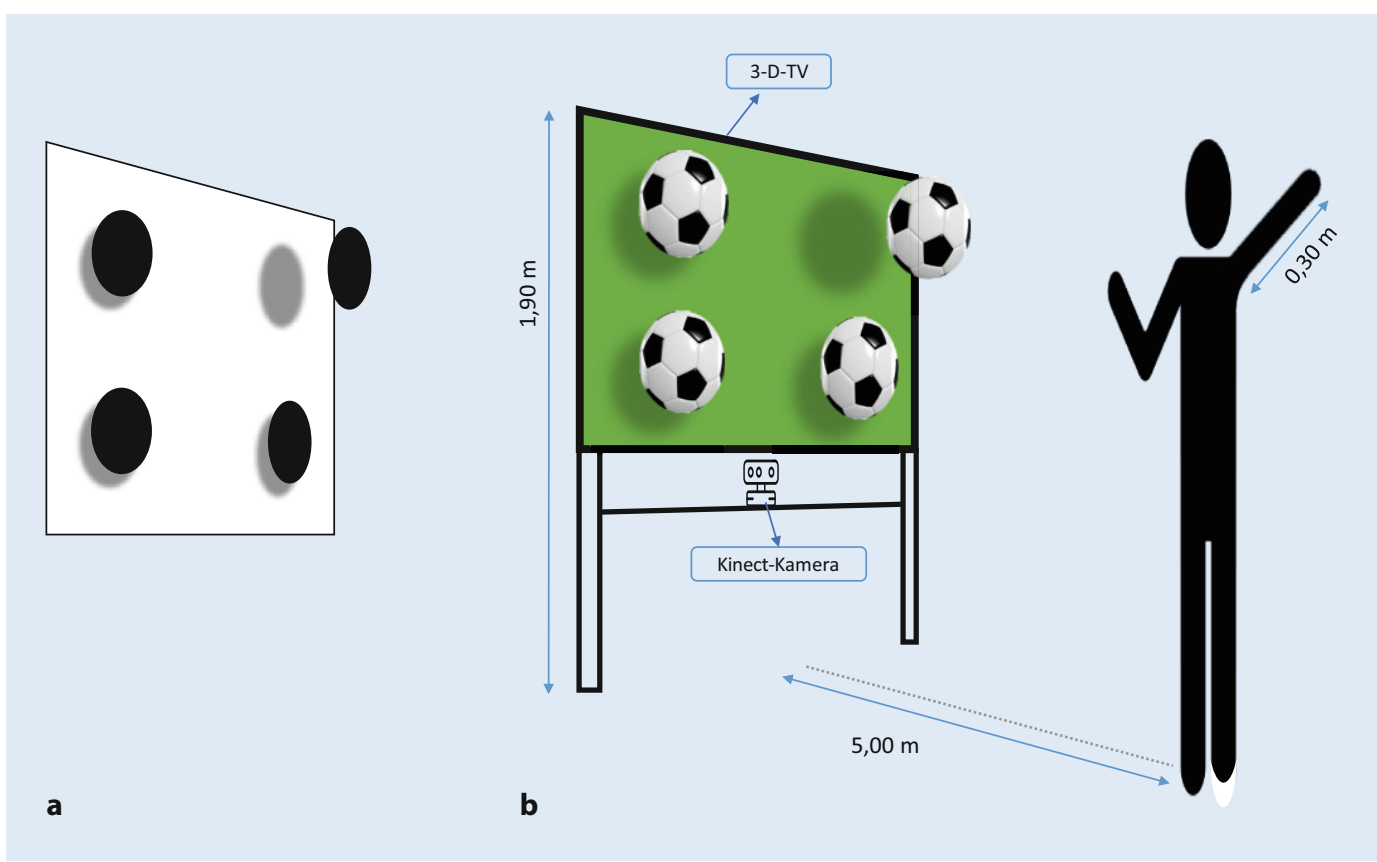

Abb. $1<$ Schematische Trainingsanordnung des c-DIGITAL VISION TRAINER (Talkingeyes \& More $\mathrm{GmbH})^{\circledR}$ bei dynamischen Stimuli mit rotierenden Bällen. a Darstellung der statischen Stimuli mit bewegungslosen Scheiben. $\mathbf{b}$ Trainingsanordnung der dynamischen Stimuli mit rotierenden Fußbällen kannte Distanzunterschied, desto besser ist das 3-dimensionale Auflösungsvermögen. Das visuelle System benötigt Zeit zur Erkennung und Beantwortung von Reizen. Je schwieriger das Sehzeichen, desto länger integrieren Neurone, um es zu erkennen. Die Reaktionszeit beinhaltet Zeitintervalle für (1) Reizaufnahme im Rezeptor (u. a. Augenbewegung, Akkommodation), (2) Übertragung zum Kortex, (3) zentrale Vorgänge (u.a. Fusion der Bilder), (4) efferente Übertragung, (5) Latenzzeit des Muskels bis zur motorischen Reaktion [8]. Für die Verarbeitung des Zeitintervalls 1 bis 3 zeigte Bergua anhand von VEP(visual evoked potentials)-Untersuchungen mit stereoskopischen Stimuli das Erscheinen von kortikalen Signalen nach $125,8 \pm 13 \mathrm{~ms}$ [9]. Beruhend auf kortikaler Plastizität [10], können wiederholte Tests stereoskopischer Stimuli bei optimalem Refraktionsausgleich [11] eine Verbesserung der Stereosehschärfe bewirken [12]. Schmitt et al. belegten anhand eines 2-Alternativen-Tests (Erkennen des vertikalen Balkens vor oder hinter einem Referenzrahmen) und variierendem, adaptivem Stereogrenzwinkel die Erlernbarkeit des Stereosehens [13].

Zur Behandlung der Amblyopie und gestörten Fusion bei anisometropen Patienten wird neben der klassischen Okklusionstherapie binokulares kontrastadaptiertes Sehtraining verwendet [14]. Dadurch können die Sehschärfe am amblyopen Auge, die Stereosehfähigkeit und die Erkennungsgeschwindigkeit verbessert werden [15]. Patienten mit Strabismus und Amblyopie profitieren auch von einem Sehtraining hinsichtlich Stereo- und Kontrastsehfähigkeit [16]. In der Presbyopietherapie konnte durch ein Wahrnehmungstraining mit niedrigen Kontrast-Gabor-Stimuli eine Verbesserung der Stereosehschärfe und visuellen Kognitionszeit erreicht werden [17].

Unsere Arbeitsgruppe zeigte in kürzlich publizierten Studien eine signifikante Verbesserung des Stereosehens bei sehgesunden Jugendlichen und Senioren durch ein auf perzeptionellem Lernen basiertes Sehtraining [18, 19].

Badminton- und Tischtennisspieler scheinen im Vergleich zu Nichtathleten zum einen eine schnellere visuelle Wahrnehmungs- und Verarbeitungsgeschwindigkeit zu erzielen und zum anderen über langjähriges Training Anpassungen im visuellen Bewegungssystem zu entwickeln, die die Wahrnehmung und Verarbeitung visueller Informationen erleichtern und folglich visuomotorische Reaktionen beschleunigen [20]. Bei Tischtennisspielern zeigte sich (mittels EMG und EEG), dass schnellere visuomotorische Reaktionen nicht auf motorische Prozesse, sondern auf visuelle Prozesse im bewegungssen- sitiven MT(mediotemporalen)-Areal des Gehirns zurückzuführen waren [20].

Bestmöglicher Erfolg im Tennissport erfordert unterschiedliche Attribute, wie z. B. hohe Oberkörperkraft [21], schmaler Körperbau, hohe Geschwindigkeit, hohe Ausdauer und Talent. Die jungen Tennisspieler an der Tennisbase in Oberhaching, ausgewählt nach möglichst vielen der genannten Attribute, erlernen in ihrer Ausbildung ähnliche grundlegende Techniken. Dennoch gibt es erhebliche Unterschiede im Tenniserfolg. Ein Teil dieses Erfolges könnte auf ein unterschiedliches Level im Stereosehen zurückzuführen sein. Dies wird auch in anderen Studien bestätigt [22]. So können z.B. Personen mit hoher Stereosehschärfe einen Tennisball besser abfangen als Personen mit geringer Stereosehschärfe [23]. Auch nach spezifischem und intensivem Training ließ sich der Nachteil im Stereosehen noch nachweisen.

Derzeit wird nur die Sehschärfe (Screeningtest) bei den jährlichen Leistungstests im DTV/BTV (Deutscher und Bayerischer Tennisverband) als Bestandsaussage der Sportler herangezogen. Obwohl die Stereosehschärfe ebenfalls ein entscheidender Faktor sein könnte, wurde sie bisher noch nicht integriert.

Wir stellten deshalb die Frage, ob Tennisspieler mit bereits gutem Stereosehen ihre Stereosehschärfe, Reaktionsgeschwindigkeit und Genauigkeit (Korrekt- 
Tab. 1 Fragebogen nach dem Training

Subjektive Auswertung des binokularen Sehtrainings

\begin{tabular}{|l|l|l|}
\hline 1 & $\begin{array}{l}\text { Das Sehtraining hat eine positive Auswirkung auf die } \\
\text { sportliche Leistung }\end{array}$ & Position auf der Skala: 3,7/5 \\
\hline 2 & Entfernungen werden besser eingeschätzt & Position auf der Skala: $3,6 / 5$ \\
\hline 3 & Das Training macht insgesamt Spaß & Position auf der Skala: 4,4/5 \\
\hline 4 & Bewertung des Trainings insgesamt & Schulnote "gut ${ }^{\prime \prime}(1,8 / 6)$ \\
\hline 5 & Kommentare/offene Fragen & - \\
\hline
\end{tabular}

heit der Versuche) anhand des binokularen Sehtrainings noch verbessern können.

\section{Studiendesign und Unter- suchungsmethoden}

Sehschärfe. Mittels des Freiburger Sehschärfetest (Fract) [24] wurde im Vorfeld die Sehschärfe beider Augen mit und ohne Sehhilfe bestimmt. Voraussetzung zum Training war ein Dezimalvisus mit Sehhilfe am schlechteren Auge von $\geq 1,0$.

Sehtraining. Der c-Digital Vision Trainer ${ }^{\circledR}$ (Talkingeyes \& More GmbH, Medical Valley Center Erlangen, Deutschland), entwickelt im Rahmen des drittmittelgeförderten Forschungsprojektes PBN-MED-16030007 von der Technischen Fakultät der Friedrich-Alexander Universität Erlangen und der Talkingeyes \& More $\mathrm{GmbH}$, ist ein digitales visuelles Wahrnehmungslernsystem, welches auf einem 3-D-Fernsehgerät einen von 4 Stimuli (Bälle/Scheiben) mit einem Tiefenunterschied präsentiert, der von einem Probanden in $5 \mathrm{~m}$ Entfernung mit Polarisationsbrille erkannt werden muss $[13,18,19]$. Im Training wählt der Proband den ihm räumlich näher erscheinenden Ball, indem er seinen Arm in Richtung des Quadranten dieses Balles streckt (• Abb. 1). DieZeitmessung wird mittels Kinect-Systems automatisch nach der Stimuluspräsentation gestartet und angehalten, sobald der Proband seine Hand $>30 \mathrm{~cm}$ von seiner Schulter entfernt bewegt. Zur Steigerung der Trainingseffektivität wurde ein positives bzw. negatives Feedback [25] gegeben, indem das Ergebnis nach jedem Versuch visuell angezeigt wurde. Für das Training wurden dynamische Stimuli verwendet, für die Messung des Trainingserfolges statische Stimuli. Durch die Unterscheidung der Stimuli wird verdeutlicht, dass eine Verbesserung der motorischen Reaktionszeit (z.B. durch die re- petitive Ausübung von Bewegungen, die zu einer optimalen Erkennung des KinectSystems führen) somit vernachlässigt werden kann bzw. nicht gemessen werden soll. Es soll die rein visuelle Reaktionszeit bzw. Kognitionszeit verbessert werden.

Messung des Trainingseffektes. Zur Überprüfung des Trainingserfolges wurden einfarbige Scheiben ohne Rotation und Textur als Stimuli verwendet (- Abb. 1). Hierbei drückt der Sportler 1/4 Knöpfe eines Kontrollers. Dies erfolgte vor, nach je 3 und 6 Trainingseinheiten.

Messparameter. Wir untersuchten für verschiedene Disparitätsdifferenzen (Bogensekunden) der Scheiben/Bälle die Reaktionszeit in ms, die Korrektheit in \% und den „ReSt" in ms/Bogensekunden (dRT/dSD).

ReSt (in ms/Bogensekunden [dRT/ dSD]). Der Parameter "ReSt" (Reaktionszeitzuwachs pro Stereodisparitätsabnahme) wurde verwendet, damit eine Verbesserung der Gesamtreaktionszeit nicht nur auf eine Verbesserung der rein motorischen Komponente zurückzuführen ist (z.B. Erlernen der optimalen Ausführung der Armbewegung mit minimalem Kraftaufwand). Dabei wird angenommen, dass der Zeitanteil für die motorische Reaktionszeit bei variierenden Disparitätsstufen gleich groß ist. Der ReSt stellt somit dasjenige Zeitintervall dar, das das visuelle System zur Bewältigung des stereoskopischen Schwierigkeitszuwachses benötigt. Zur Berechnung wurde die Differenz der Reaktionszeit bei Stimuli von 15 und 45 Bogensekunden verwendet. Der ReSt stellt damit ein Maß für die rein visuelle Kognitionszeit dar und ist somit unabhängig von der motorischen Fähigkeit und Schnelligkeit.
Trainingsablauf. Die Probanden absolvierten 7 bis 13 Trainingseinheiten (mindestens 2/Woche über mindestens 5 Wochen, „mean“ 8, maximal 12 Wochen) à 192 visuellen Trainingsaufgaben und damit insgesamt mindestens 1602 Einzelaufgaben. Die Anzeige der stereoskopischen Stimuli erfolgte nach der Methode der konstanten Reize (Konstanzmethode) [26]. Es wurde eine Serie von 6 Disparitätsstufen (15; 30; 45; 60; 150; 300 Bogensekunden) in randomisierter Reihenfolge gezeigt. Jede Disparitätsstufe wurde 17-mal gezeigt, wobei der erste Versuch pro Disparitätsstufe nicht gewertet wurde. Um die jeweilige Disparität als erkannt zu werten, mussten mindestens 10 von 16 Stimuli (Ratewahrscheinlichkeit $<0,01 \%)$ des geprüften Disparitätsunterschiedes korrekt erkannt werden. Der Stereogrenzwinkel wurde als Maß für die Stereosehschärfe angegeben [27].

Probanden. Getestet wurden 31 Probanden (26 männlich; 5 weiblich), die zum Zeitpunkt der Untersuchung in unterschiedlicher Form im Bereich Tennis tätig waren (14 Jugendspieler beim Bayerischen Tennis-Verband, 6 Vollprofis, 10 Trainer bzw. ehemalige Vollprofis).

Fragebogen. Alle Probanden wurden gebeten, anhand eines Fragebogens (- Tab. 1) die subjektive Wirkung des Sehtrainings zu evaluieren. Der Fragebogen bestand aus 4 Fragen, die mittels Likert-Skala von 1 bis 5 (trifft nicht zu - trifft zu) zu bewerten waren, 2 offenen Fragen (freies Textfeld) und einer Frage mit Schulnotenvergabe von 1 bis 6 (sehr gut - ungenügend).

\section{Ergebnisse}

Trainingseffekte, gemessen mit texturfreien und bewegungslosen Scheiben.

Durch das binokulare Sehtraining kam es bei Tennisspielern zu einer signifikanten Verbesserung der Stereosehschärfe, Reaktionszeit und Korrektheit. Bei der Berechnung des Signifikanzniveaus wurde multiples Testen berücksichtigt. Somit ergibt sich für diese Tests ein neues Signifikanzniveau von $1 \%(0,01)$.

Die mittlere Reaktionszeit verlängerte sich mit Zunahme des Schwierigkeitsgra- 


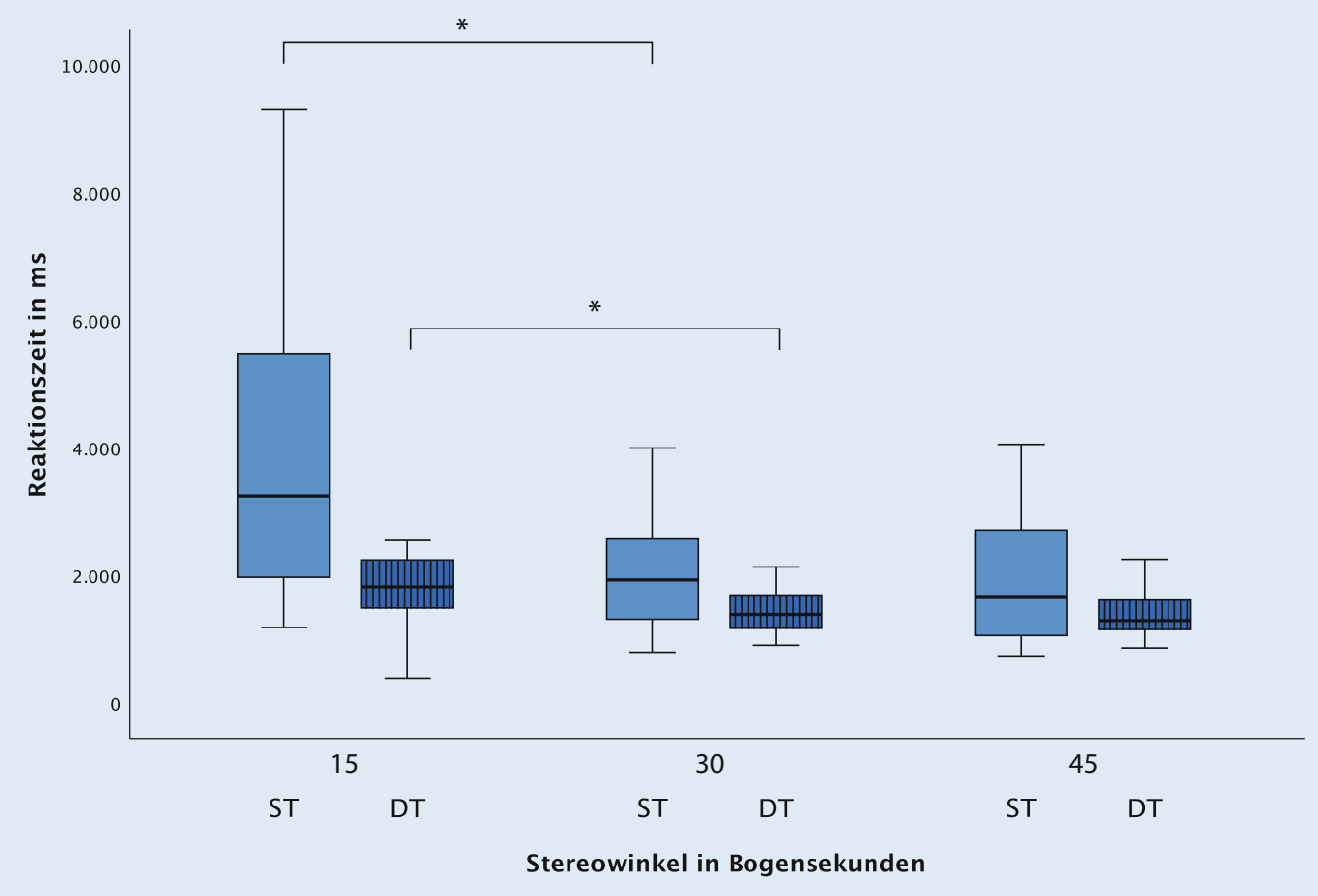

Abb. $2 \triangleleft$ Boxplot der Reaktionszeiten aller Probanden auf verschiedenen Stufen (15 Bogensekunden, 30 Bogensekunden, 45 Bogensekunden) bei statischen Stimuli (ST) und bei dynamischen Stimuli (DT) vor dem Training. Die Reaktionszeit bei dynamischen Stimuli war signifikant kürzer als bei statischen Stimuli. Asterisk signifikant $(p<0,05)$

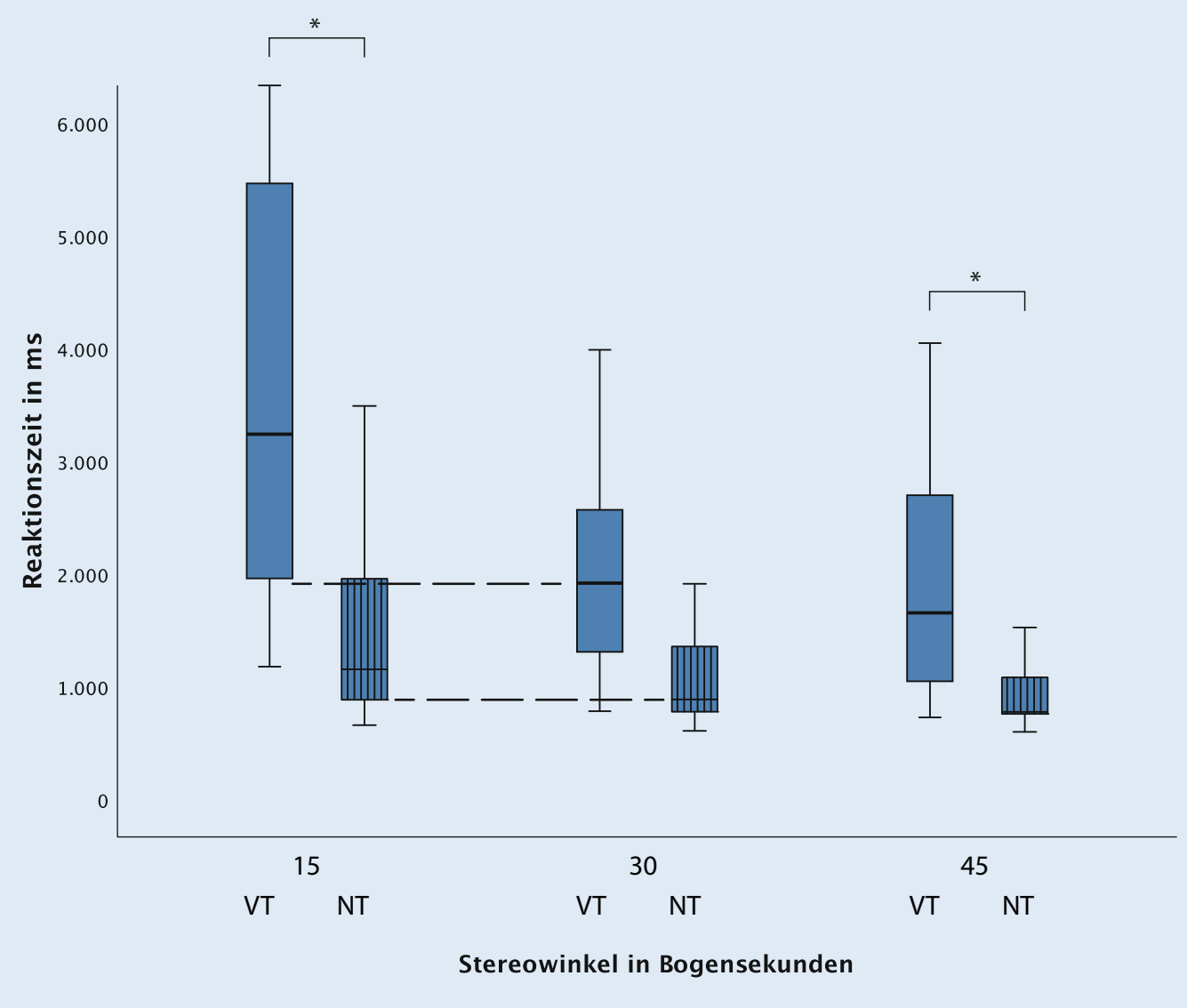

Abb. $3 \triangleleft$ Trainingseffekt: Boxplot der Reaktionszeiten aller Probanden auf verschiedenen Stufen (15, 30, 45 Bogensekunden) bei statischen Stimuli vor dem Training (VT) und nach dem Training (NT). Asterisk signifikant $(p<0,01)$ 


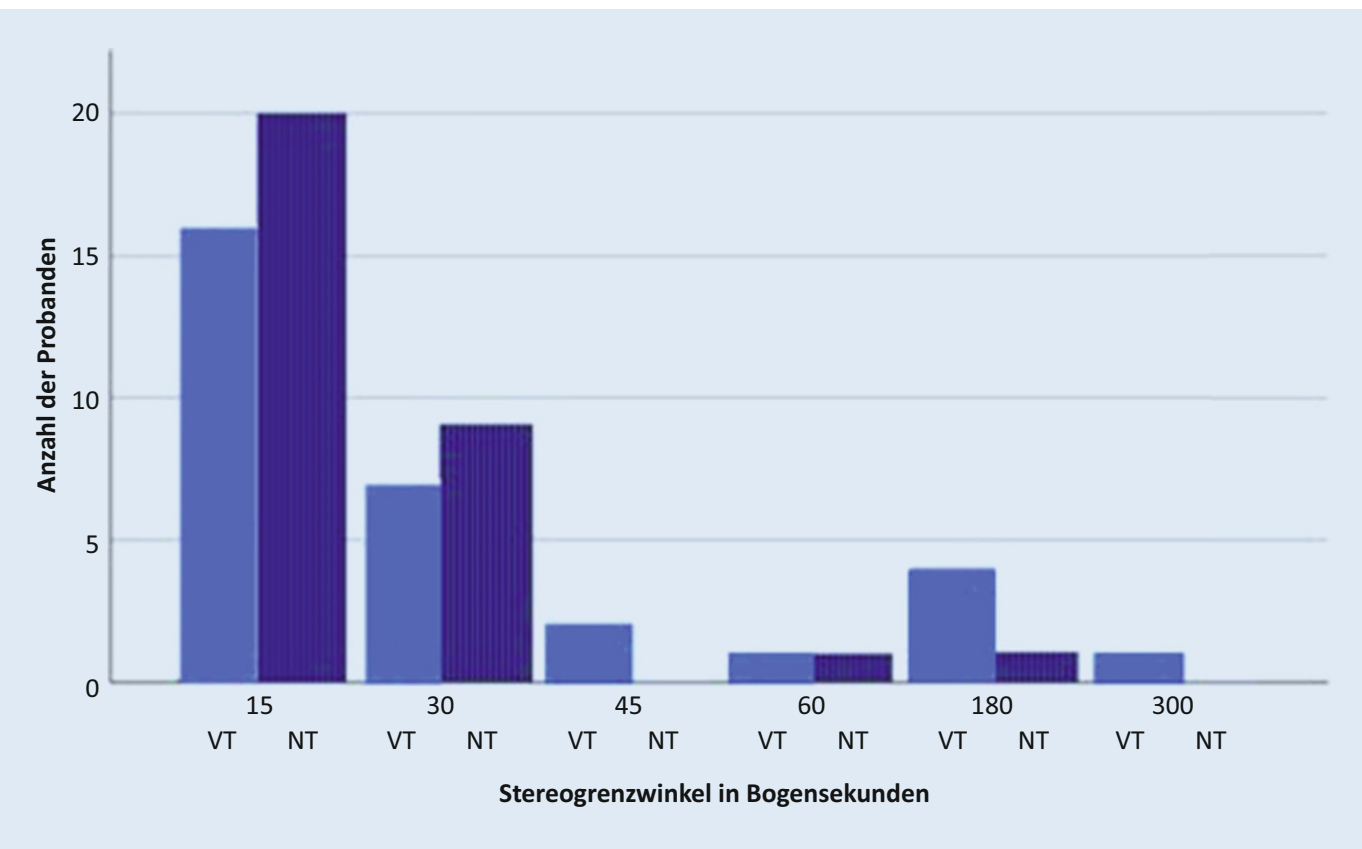

Abb. $4 \varangle$ Verteilung des Stereogrenzwinkels bei statischen Stimuli vor dem Training $(V T)$ und nach dem Training (NT)

des (der Disparitätsdifferenz) (• Abb. 2). Die Reaktionszeit für 15 Bogensekunden war im Mittel 3,9s und signifikant länger als die Reaktionszeit von $2,7 \mathrm{~s}$ für $30 \mathrm{Bo}$ gensekunden $(p=0,0001)$.

Alle Probanden verbesserten sich signifikant hinsichtlich der Reaktionszeit bei Stimuli von 45 Bogensekunden von $2,3 \mathrm{~s} \pm 0,4 \mathrm{~s}$ auf $1,1 \mathrm{~s} \pm 0,1 \mathrm{~s} \quad(p=0,0003)$ (- Abb. 3); 28/31 Probanden verbesserten sich hinsichtlich der Reaktionszeit bei Stimuli von 15 Bogensekunden, im Mittel kam es zu einer signifikanten Verbesserung von $59 \%$ von $3,9 \mathrm{~s} \pm 0,5 \mathrm{~s}$ auf $1,6 s \pm 0,2 s(p=0,00002)$.

Vor dem Training erreichten $74 \%$ der Sportler einen Stereogrenzwinkel von 15 oder 30 Bogensekunden (16/31 Probanden davon 15 Bogensekunden), nach dem Training 93,5\% (- Abb. 4). $N=13$ von $15(87 \%)$ Athleten, die in der Eingangsmessung einen Stereogrenzwinkel von 30 Bogensekunden oder schlechter aufwiesen, zeigten eine Verbesserung des Stereogrenzwinkels um mindestens 1 Stufe, die anderen beiden blieben konstant bei 30 Bogensekunden. Der Median dieser Probanden verbesserte sich signifikant von 45 auf 15 Bogensekunden $(p=0,001)$ (• Abb. 5). - Abb. 6 zeigt die Verbesserung des Stereogrenzwinkels in Abhängigkeit von der Reaktionszeit für die Probanden, die in der Eingangsmessung nicht schon maximal erreichbare
15 Bogensekunden aufwiesen. Für eine optimale Reaktionszeit bei optimalem Stereogrenzwinkel muss die Position des Probanden so nah wie möglich am Ursprung der Abbildung sein. Die Verteilung der Probanden im Abschlusstest erscheint deutlich ursprungsnäher als im Eingangstest.

Der ReSt verminderte sich durch das Training signifikant von $1,6 \mathrm{~s} \pm 0,2 \mathrm{~s}$ auf $0,5 \mathrm{~s} \pm 0,2 \mathrm{~s}(67 \%)(p=0,002) \quad(\cdot$ Abb. 7); $83,9 \%$ der Probanden konnten ihren individuellen ReSt verbessern (darunter 61,9\% um über $1000 \mathrm{~ms}$ ). Die • Abb. 7 zeigt die Verkürzung des Zeitintervalls, das für das visuelle System nötig ist, um den stereoskopischen Schwierigkeitszuwachs von 45 zu 15 Bogensekunden zu bewältigen.

Auch die Erkennenssicherheit verbesserte sich bei einem Stereogrenzwinkel von 15 und 30 Bogensekunden. Vor dem Training waren bei 30 Bogensekunden im Mittel $36 \%$ der Versuche fehlerhaft, nach dem Training nur noch $13 \%$ (signifikante Verbesserung um $23 \%[p=0,0004])$.

Vergleich des Trainingseffektes bei bewegungslosen Scheiben und rotierenden Bällen als Stimuli. Insgesamt zeigte sich im Trainingsverlauf eine größere Verbesserung des Stereogrenzwinkels im dynamischen Test (um 61,3\%, bei statischen Stimuli um 41,9\%), sodass hier nach dem Training 74,2\% der Probanden einen Stereogrenzwinkel von 15 Bogensekunden aufweisen konnten.

Die Reaktionszeiten beim statischen und dynamischen Test sind signifikant unterschiedlich. Bei statischen Tests verkürzte sich die Reaktionszeit im Mittel bei 15 Bogensekunden-Stimuli signifikant um $59 \%(p=0,00002)$, im dynamischen Training signifikant um 30,5\% (von 2,1 s auf $1,4 s)(p=0,023)$. Die Reaktionszeitverbesserung stieg mit dem Schwierigkeitsgrad der Stimuli bei beiden Tests an (am höchsten bei 15 Bogensekunden). Die Verbesserung des ReSt war im statischen Test (67\%) größer als bei dynamischen Stimuli. Im dynamischen Training kam es bei 22/31 Probanden im Mittel zu einer signifikanten Verbesserung der ReSt um $53 \%$ (von $0,7 \mathrm{~s}$ auf $0,3 \mathrm{~s})(p=0,046)$.

Fragebogen. Insgesamt wurde die Aussage Sehtraining hat eine eher positive Auswirkung auf die sportliche Leistung mit 3,7/5 bewertet. Probanden mit einer Verbesserung des Stereogrenzwinkels $>30$ Bogensekunden bewerteten die Aussage im Mittel mit 4,0/5 und Probanden mit einer Verbesserung der Stereogrenzwinkelreaktionszeit >200 ms bei konstant bleibenden 15 Bogensekunden mit 4,3/5. Die Aussage Entfernungen können besser eingeschätzt werden wurde mit 3,6/5 bewertet; $73 \%$ der Tennisspieler jünger als 17 Jahre kommentierten, dass sie in 


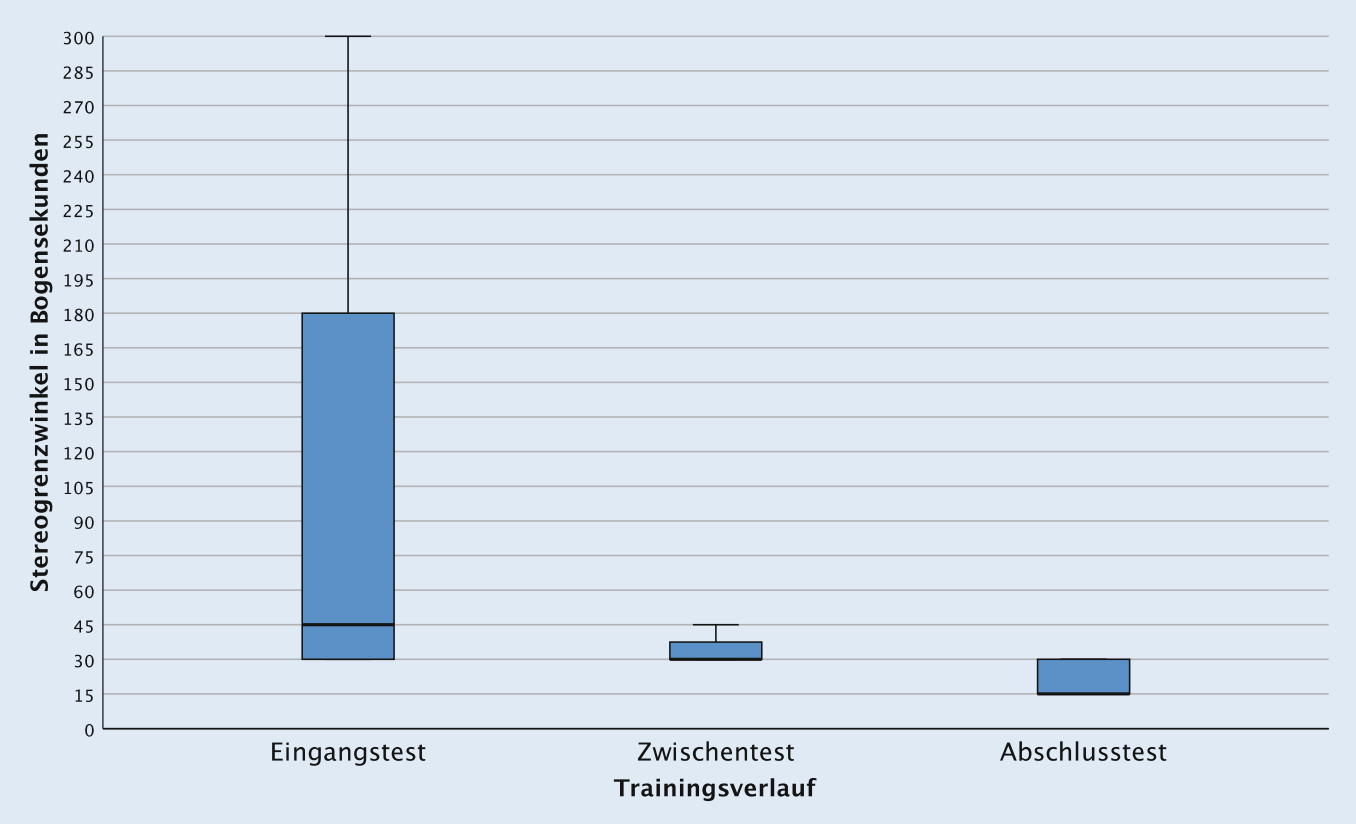

Abb. 54 Stereogrenzwinkel im Trainingsverlauf bei statischen Stimuli für Probanden $>15$ Bogensekunden in der Eingangsmessung

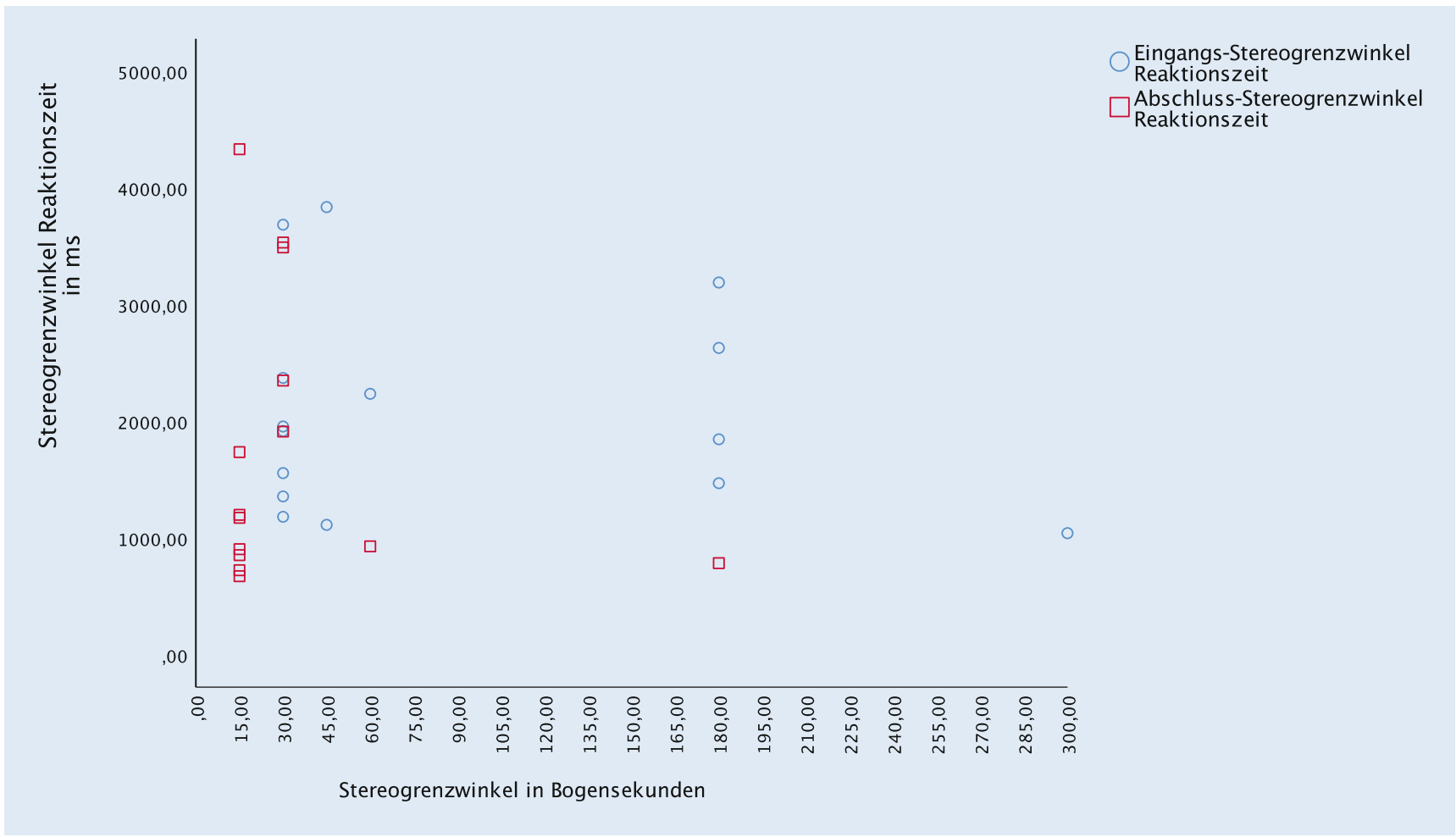

Abb. 6 \ Reaktionszeitverbesserung in Abhängigkeit der Stereogrenzwinkelverbesserung für Probanden > 15 Bogensekunden in der Eingangsmessung bei statischen Stimuli

der Schule besser aufpassen und insgesamt das Tafelbild besser erkennen und verstehen können. Die Aussage Das digitale Sehtraining machte Spaß wurde mit $4,4 / 5$ und das Sehtraining insgesamt mit der Schulnote "gut" $(1,8 / 6)$ bewertet.

\section{Diskussion}

Da im Testsystem die höchste Stufe bei maximal 15 Bogensekunden gewählt wurde, war es bei der Hälfte der Probanden nicht möglich, eine Verbesserung des Stereogrenzwinkels nachzuweisen. Deshalb wur- den Parameter wie Reaktionszeit und Korrektheit bestimmt, so konnte auch in dieser Gruppe eine Verbesserung im Stereosehen erreicht werden.

Die Analyse der Erkennenssicherheit im Trainingsverlauf zeigte, dass die Verbesserung der Reaktionszeit insgesamt nicht zur 


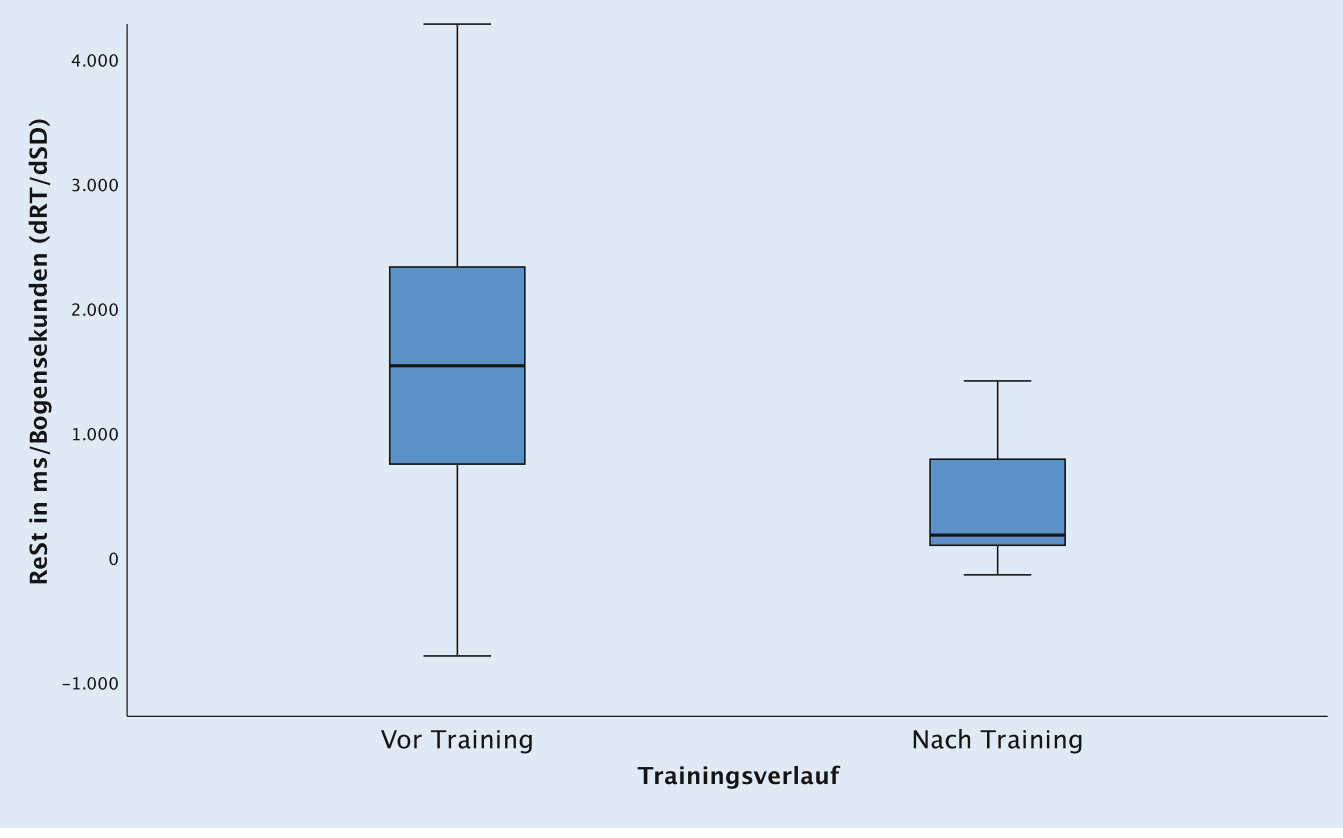

Abb. $7 \varangle$ Reaktionszeitzuwachs pro Stereodisparitätsabnahme in ms/Bogensekunden $(d R T / d S D)$ vor und nach dem Training bei statischen Stimuli

Abnahme der Korrektheit der erkannten Stimuli führte. Über die Hälfte der Probanden (65\%) konnte ihre Erkennenssicherheit bei 30 Bogensekunden im Mittel um ca. ein Viertel (23\%) verbessern. In • Abb. 6 lässt sich anhand der Verteilung der Punkte erkennen, dass die Verbesserung der Reaktionszeit insgesamt nicht mit einer Verschlechterung des Stereogrenzwinkels einhergeht.

Die Probanden hatten zu Beginn des Trainings eine interindividuell variierende Vorerfahrung sowohl in psychophysikalischen Experimenten als auch im Umgang mit technischen Messsystemen (z. B. Kinect-System oder Kontroller). Diese Erfahrung ist schwer einzuordnen. Die Arbeit von Schmitt zeigt jedoch, dass es für die Messung bzw. das Training der Stereosehschärfe keinen signifikanten Unterschied zwischen Anfängern und erfahrenen Probanden gibt [13].

Um die kognitiven Effekte von Verbesserungen der Motorik durch wiederholtes Testen zu unterscheiden, muss auch das Lernverhalten der einzelnen Probanden berücksichtigt werden. Bei interindividueller Analyse der Probanden lassen sich verschiedene Lerntypen (z. B. Nichtlerner und Erlerner) erkennen. Durch den Zwischentest nach 3 Trainingseinheiten konnten wir zwischen Langsam- und Schnelllernern unterscheiden. Somit ist der Beginn einer adäquaten Trainingsphase ein wichtiger
Faktor. In einigen Studien wird beschrieben, dass die Lerneffekte auf die Art des für das Training verwendeten Ziels beschränkt sind [13]. Durch die Trennung der sensorischen Reize (Trainingsstimuli/dynamische Stimuli von Teststimuli/statische Stimuli) wird jedoch eine Transferaufgabe geleistet, die es erlaubt, die motorische Verbesserung zu vernachlässigen. Um die kurzfristige motorische Verbesserung („warm up ${ }^{\prime \prime}$ ) vernachlässigen zu können, wurde vor jedem Training und Test jede Schwierigkeitsstufe einmal präsentiert. Das Ergebnis wurde nicht aufgezeichnet.

Es zeigte sich, dass am Anfang des Trainings die stärksten Verbesserungen zu erkennen waren. Nach dem dritten Trainingsset konnte keine relevante Verbesserung mehr gezeigt werden. Unsere Beobachtung, dass Verbesserungen der Stereosehschärfe nach einer gewissen Trainingszeit ein Plateau erreichen, wird von anderen Studien $[18,19]$ bestätigt.

Optimale Stereosehschärfe kann für den Ablauf im Tennisspiel von wichtiger Bedeutung sein. Das räumlich-visuelle Sehsystem kombiniert anhand von Fixpunkten (z.B. das Netz, die Bodenlinien etc.) Details, wie z.B. die Ballform, Balldrehung, Ballschatten, Aufprallwinkel, Aufprallgeschwindigkeit, und Stellung bzw. Schlag des Gegenspielers, um eine genaue Flugbahn des ankommenden Balles zu berechnen. Abhängig von der
Berechnung des Balles, kann der Spieler reagieren, um den Ball wieder zurückspielen zu können. Shim et al. bestätigte bessere Antizipationsfähigkeiten von professionellen Tennisspielern gegenüber Anfängern [28].

Eine Verknüpfung von kortikalen multisensorischen Bereichen könnte ebenfalls zu einer Verbesserung des Tennisspiels führen. Akustische Reize, wie z.B. der Aufprall des Balles am Schläger des Gegenspielers, geben Aufschluss über Härte und Drall des Schlages. Unter repetitiver Präsentation kann das Geräusch die Empfindlichkeit der visuellen Wahrnehmung erhöhen [29] und die Qualität im Tennisspiel steigern.

Die Auswirkung des Sehtrainings auf die sportliche Leistung ist schwer zu messen. Anhand des Fragebogens konnte jedoch gezeigt werden, dass sich die Selbsteinschätzung im Sport auch im Ergebnis des Sehtrainings widerspiegelt. So bewerteten Probanden, die sich im Sehtraining deutlich verbesserten (Verbesserung Stereogrenzwinkel $>30$ Bogensekunden bzw. Verbesserung Stereogrenzwinkelreaktionszeit $>200 \mathrm{~ms}$ bei konstanten 15 Bogensekunden), auch die Aussage Sehtraining hat eine eher positive Auswirkung auf die sportliche Leistung im Mittel besser (mit 4,0/5 bzw. 4,3/5) als der Rest der Probanden (mit 3,7/5 bzw. $3,5 / 5)$. 


\section{Schlussfolgerung}

Unsere Studienergebnisse zeigen, dass es bei Hochleistungssportlern mit guter Stereosehschärfe durch binokulares Sehtraining zu einer signifikanten Verbesserung der Stereosehschärfe, Reaktionszeit und Korrektheit der Antworten kommt.

Bei Senioren und jungen Fußballspielern konnten bereits ähnlich erfolgreiche Ergebnisse mit dem c-Digital Vision Trainer $^{\circledR}$ erzielt werden. Bei 15 jungen Fußballspielern kam es nach 6 Trainingseinheiten mit unterschiedlichen Disparitätsdifferenzen (11 bis 88 Bogensekunden) zu einer signifikanten Abnahme der mittleren Reaktionszeit bei 11 Bogensekunden von $2524 \mathrm{~ms}$ auf $869,5 \mathrm{~ms}$. Auch die Reaktionszeit bei 88 Bogensekunden verkürzte sich signifikant [18]. Elf Senioren (Durchschnittsalter: 85,90 Jahre) konnten ihren Stereogrenzwinkel über 6 Wochen Training signifikant von 138 auf 69 Bogensekunden verbessern [19].

Die Auswirkungen der Verbesserungen im Stereosehen auf den Sport bzw. auch den Alltag der Probanden sind jedoch abschließend auch durch den Fragebogen nicht eindeutig und neutral geklärt. Hierbei benötigt es weitere Analysen und Studien.

Ein zukünftiger Ansatz für die Messung der sportlichen Relevanz könnte hierbei z. B. ein mit Kameras und Sensoren ausgestatteter Tennisschläger sein. Dieser könnte u.a. Aufprallwinkel, -ort und -kraft vom Tennisball auf den Schläger vor und nach dem Training messen.
Korrespondenzadresse

\section{Benedikt L. Kutzner}

Department of Ophthalmology, FriedrichAlexander-Universität Erlangen-Nürnberg (FAU)

Schwabachanlage 6, 91054 Erlangen,

Deutschland

benedikt.kutzner@hotmail.de

\section{Dr.-Ing. Matthias Ring}

Department Artificial Intelligence in Biomedical Engineering (AIBE), Lehrstuhl für Maschinelles Lernen und Datenanalytik, FriedrichAlexander-Universität Erlangen-Nürnberg (FAU) Carl-Thiersch-Str. 2b, 91052 Erlangen, Deutschland matthias.ring@fau.de

\section{Prof. Dr. Georg Michelson}

Department of Ophthalmology, FriedrichAlexander-Universität Erlangen-Nürnberg (FAU) Schwabachanlage 6, 91054 Erlangen, Deutschland georg.michelson@uk-erlangen.de

Danksagung. Die Autoren danken dem Deutschen und Bayerischen Tennis-Verband für ihre hervorragende Zusammenarbeit. Bei dieser Publikation handelt es sich um eine Promotionsleistung zur Erlangung des Doktors der Medizin (Dr. med.) an der Friedrich-Alexander-Universität Erlangen-Nürnberg (FAU).

Funding. Open Access funding enabled and organized by Projekt DEAL.

\section{Einhaltung ethischer Richtlinien}

Interessenkonflikt. B. Kutzner, M. Ring und G. Michelson geben an, dass kein Interessenkonflikt besteht. Uns sind keine Interessenkonflikte im Zusammenhang mit dieser Veröffentlichung bekannt. Es gab keine nennenswerte finanzielle Unterstützung für diese Arbeit, die ihr Ergebnis beeinflusst haben könnte. Die Software des c-Digital Vision Trainer wurde im Rahmen eines drittmittelgeförderten Forschungsprojektes PBN-MED-1603-0007 von der Technischen Fakultät der Friedrich-Alexander Universität Erlangen und der Talkingeyes \& More GmbH entwickelt. G. Michelson ist Eigentümer der Talkingeyes \& More GmbH. Als korrespondierender Autor bestätige ich, B. Kutzner, dass das Manuskript von allen genannten Autoren gelesen und zur Einreichung freigegeben wurde.

Alle beschriebenen Untersuchungen am Menschen wurden mit Zustimmung der zuständigen Ethik-Kommission, im Einklang mit nationalem Recht sowie gemäß der Deklaration von Helsinki von 1975 durchgeführt. Dieser Beitrag beinhaltet keine Studien an Menschen oder Tieren.

Open Access. Dieser Artikel wird unter der Creative Commons Namensnennung 4.0 International Lizenz veröffentlicht, welche die Nutzung, Vervielfältigung, Bearbeitung, Verbreitung und Wiedergabe in jeglichem Medium und Format erlaubt, sofern Sie den/die ursprünglichen Autor(en) und die Quelle ordnungsgemäß nennen, einen Link zur Creative Commons Lizenz beifügen und angeben, ob Änderungen vorgenommen wurden.

Die in diesem Artikel enthaltenen Bilder und sonstiges Drittmaterial unterliegen ebenfalls der genannten Creative Commons Lizenz, sofern sich aus der Abbildungslegende nichts anderes ergibt. Sofern das betreffende Material nicht unter der genannten Creative Commons Lizenz steht und die betreffende Handlung nicht nach gesetzlichen Vorschriften erlaubt ist, ist für die oben aufgeführten Weiterverwendungen des Materials die Einwilligung des jeweiligen Rechteinhabers einzuholen.

Weitere Details zur Lizenz entnehmen Sie bitte der Lizenzinformation auf http://creativecommons.org/ licenses/by/4.0/deed.de.

\section{Literatur}

1. Morris D, Fraser SG, Gray C (2007) Cataract surgery and quality of life implications. Clin Interv Aging 2(1):105-108. https://doi.org/10.2147/ciia.2007. 2.1.105

2. Gresset J, Meyer F (1994) Risk of accidents among elderly car drivers with visual acuity equal to $6 / 12$ or $6 / 15$ and lack of binocular vision. Ophthalmic Physiol Opt 14(1):33-37. https://doi.org/10.1111/ j.1475-1313.1994.tb00553.x

3. Coleman A, Cummings S, Yu F et al (2007) Binocular visual-field loss increases the risk of future falls in older white women. J Am Geriatr Soc 55(3):357-364. https://doi.org/10.1111/j.15325415.2007.01094.x

4. Faubert J (2013) Professional athletes have extraordinary skills for rapidly learning complex and neutral dynamic visual scenes. Sci Rep. https:// doi.org/10.1038/srep01154

5. Optometry \& Visual Performance Peripheral awareness and visual reaction time in professional football players in the national football league (N.F.L.). www.vision-institute.com/wp-content/ uploads/2018/03/Peripheral-Awareness-andVisual-Reaction-Time-in-Professional-FootballPlayers-in-the-National-Football-League-NFL. pdf.Zugegriffen: 3. Febr. 2021

6. Beals RP, Mayyasi AM, Templeton AE et al (1971) The relationship between basketball shooting performance and certain visual attributes. Am J Optom Arch Am Acad Optom 48(7):585-590. https://doi.org/10.1097/00006324-19710700000007

7. Vera J, Molina R, Cárdenas D, Redondo B, Jiménez R (2019) Basketball free-throws performance depends on the integrity of binocular vision. Eur J Sport Sci 28:1-8

8. Pfeifer R (2015) Arsmartialis.com. http://www. arsmartialis.com/index.html?name=http. Zugegriffen:26. Sept. 2021

9. Bergua A, Horn FK, Martus P et al (2004) Stereoscopic visual evoked potentials in normal subjects and patients with open-angle glaucomas. Graefes Arch Clin Exp Ophthalmol 242:197-203. https://doi.org/10.1007/s00417-003-0797-3

10. Polat U (2014) Perceptual learning induces neuroplasticity, enabling improvement of visual functions. Expert Rev Ophthalmol 4(6):573-576. https://doi.org/10.1586/eop.09.54 
11. Saladin JJ (2005) Stereopsis from a performance perspective. Optom Vis Sci 82(3):186-205. https:// doi.org/10.1097/01.opx.0000156320.71949.9d

12. Xi J, Jia W, Feng L et al (2014) Perceptual learning improves stereoacuity in amblyopia. Invest Ophthalmol Vis Sci 55(4):2384-2391. https://doi. org/10.1167/iovs.13-12627

13. Schmitt C, Kromeier M, Bach M et al (2002) Interindividual variability of learning in stereoacuity. Graefes Arch Clin Exp Ophthalmol 240(9):704-709. https://doi.org/10.1007/s00417-002-0458-y

14. Levi D, Li R (2009) Perceptual learning as a potential treatment for amblyopia: a mini-review. Vision Res 49(21):2535-2549

15. Chen P, Chen J, Fu J (2008) A pilot study of anisometropic amblyopia improved in adults and children by perceptual learning: an alternative treatment to patching. Ophthalmic Physiol Opt 28(5):422-428

16. Shuai L, Leilei Z, Wen W et al (2019) Binocular treatment in adult amblyopia is based on parvocellular or magnocellular pathway. Eur J Ophthalmol. https://doi.org/10.1177/1120672119841216

17. Sterkin A, Levy Y, Pokroy R et al (2018) Vision improvement in pilots with presbyopia following perceptual learning. Vision Res 152:61-73

18. Schoemann M, Lochmann M, Paulus J et al (2017) Repetitive dynamic stereo test improved processing time in young athletes. Restor Neurol Neurosci. https://doi.org/10.3233/RNN-170729

19. Erbes S, Michelson G (2021) Stereoscopic visual perceptual learning in seniors. Geriatrics 6(3):94. https://doi.org/10.3390/geriatrics6030094

20. Hülsdünker T, Ostermann M, Mierau A (2019) The speed of neural visual motion perception and processing determines the visuomotor reaction time of young elite table tennis athletes. Front Behav Neurosci. https://doi.org/10.3389/fnbeh. 2019.00165

21. Kramer T, Huijgen B, Elferink-Gemser Met al (2017) Prediction of tennis performance in junior elite tennis players. J Sports Sci Med 16(1):14-21

22. Aarau K (2019) Sports vision based tennis player training. Intelligent computing, S 1193-1201 https://doi.org/10.1007/978-3-030-22868-2_83

23. Mazyn L, Lenoir M, Montagne G et al (2007) Stereo vision enhances the learning of a catching skill. Exp Brain Res 179:723-726

24. Bach M(2021) Freiburg vision test ('FrACT'). https:// michaelbach.de/fract/.Zugegriffen: 5. Apr. 2021

25. Heinrich SP, Krüger K, Bach M (2011) The dynamics of practice effects in an optotype acuity task. Graefes Arch Clin Exp Ophthalmol 249(9):1319-1326. https://doi.org/10.1007/s00417-011-1675-z

26. Lexikon der Psychologie Schwellenbestimmung. https://www.spektrum.de/lexikon/psychologie/ schwellenbestimmung/13719.Zugegriffen:5.Apr. 2021

27. Lexikon der Optik Stereowinkel. https://www. spektrum.de/lexikon/optik/stereowinkel/3212. Zugegriffen:26. Sept. 2021

28. Shim J, Carlton LG, Chow JW et al (2005) The use of anticipatory visual cues by highly skilled tennis players. J Mot Behav. https://doi.org/10.3200/ jmbr.37.2.164-175

29. Pooresmaeili A, Fitzgerald THB, Bach DRetal (2014) Cross-modal effects of value on perceptual acuity and stimulus encoding. Proc Natl Acad Sci U S A 111(42):15244-15249. https://doi.org/10.1073/ pnas. 1408873111

\section{Binocular vision training for professional athletes}

Background: Optimal visual abilities including stereo acuity seem to be an important issue in sports. There is increasing evidence that stereo acuity can be sustainably improved by digital vision training even for people with good stereo acuity.

Study design and test methods: In this study 31 male and female tennis players (professionals, young professionals, coaches and former professionals) completed at least 6 training units each with 192 dynamic stereoscopic tasks $(N=1152)$ within 6 weeks including a 4-option test with different levels of difficulty on a 3D screen at a distance of $5 \mathrm{~m}$. The parameter reaction time and correctness at 15-300 arcseconds was determined. For a more precise representation of the reaction time improvement as a function of the difficulty level, the parameter reaction time increase per stereo disparity reduction (ReST) was defined.

Results: Reaction time to 15 arcsecond stimuli significantly decreased from $3.9 \mathrm{~s}$ to $1.6 \mathrm{~s}(59 \%)$ as a result of digital vision training. The correctness at 30 arcsecond stimuli significantly increased by $23 \%$.

Discussion: The observed improvement in reaction time during vision training did not result in decreasing correctness when answering the visual questions. This represents an overall improvement in stereo vision.

Conclusion: Dynamic visual training over 6 weeks improves stereoscopic performance including stereo acuity, response time and correctness.

\section{Keywords}

Stereo vision $\cdot$ Vision training $\cdot$ Depth perception $\cdot$ Sport $\cdot$ Cognitiontime 